\title{
Propuesta de un sistema de evaluación del desempeño para una institución que brinda servicios académicos en la ciudad de Tacna
}

\section{Proposal of a performance evaluation system for an institution that offers academic services in the city of Tacna}

\author{
${ }^{1}$ Jehovanni Velarde Molina, ${ }^{2}$ Leonardo Ticona Gómez, ${ }^{3}$ Pablo Vásquez Espinoza, ${ }^{4}$ Ben \\ Yabar Vega
}

\section{RESUMEN}

El presente artículo tuvo como objetivo orientar la actuación técnica y/o profesional consiguiendo la mayor adecuación posible de las personas en sus puestos de trabajo, proponiendo un sistema de evaluación del desempeño para las escuelas educativas, en este caso se aplicó a la Escuela de Negocios John Von Neumann SRL de Tacna. Para poder conocer la evaluación de desempeño se aplicó un test de autoevaluación del desempeño laboral. Este sistema de autoevaluación de desempeño tuvo los siguientes indicadores: la perspectiva, la equidad, la fortaleza y el equilibrio. Se desarrolló la propuesta de un modelo para la evaluación del desempeño laboral del personal, basado en un conjunto de competencias generales para un desempeño laboral destacado (independiente del puesto de trabajo); dichos factores de evaluación fueron: conocimiento del trabajo, calidad de trabajo, eficiencia / productividad, actitud e iniciativa, atención al cliente y responsabilidad, solución de problemas, planificación y organización del trabajo, toma de decisiones, liderazgo y trabajo en equipo, entrenamiento y capacitación del personal. Los beneficios esperados de la implementación de la propuesta fueron conseguir que su personal se encuentre más comprometido, incrementar nivel de satisfacción laboral, tendencia a fortalecer el trabajo en equipo, mejor comunicación organizacional; además de permitir a los directivos fortalecer las capacidades de su personal.

Palabras claves: Capacidades, equidad, equilibrio, fortaleza, perspectiva, satisfacción laboral.

\section{ABSTRACT}

The aim of this article is to guide technical and/or professional action by achieving the best possible adaptation of people in their jobs by proposing a performance evaluation system for educational schools; in this case, it was applied to the Business School John von Neumann SRL of Tacna. To be able to know the evaluation of performance, a self-evaluation test of the labor performance was applied. This performance self-evaluation system has the following indicators: perspective, equity, strength and balance. The proposal of a model for the evaluation of the work performance of the personnel was developed, which is based on a set of general competences for an outstanding work performance (independent of the job position). These assessment factors are: knowledge of work, quality of work, efficiency/productivity, attitude and initiative, customer service and responsibility, problem solving, planning and organization of work, decision making, leadership and teamwork, training and Staff training. The benefits expected from the implementation of the proposal are to ensure that your staff is more committed, higher level of job satisfaction, a tendency to strengthen teamwork, better organizational communication, among others; also it allows managers to strengthen the capabilities of their staff.

Keywords: Equity, perspective, balance, strength, work satisfaction, capabilities.

\footnotetext{
'Facultad de Ciencias Empresariales y Educación. Universidad Alas Peruanas. Tacna-Perú. E-mail:j_velarde_m@doc.uap.edu.pe ${ }^{2}$ Facultad de Contabilidad y Finanzas. Universidad Latinoamericana Cima. Tacna-Perú. E-mail: 1ticona@ulc.edu.pe

${ }^{3}$ Facultad de Ciencias Empresariales y Educación. Universidad Alas Peruanas. Tacna-Perú. E-mail:p_vasquez_es@doc.uap.edu.pe

${ }^{4}$ Escuela de Postgrado Neumann Business School. Tacna-Perú. E-mail: byabar@nbs.edu.pe
} 


\section{INTRODUCCIÓN}

El presente artículo pretende aportar un sistema de evaluación de desempeño, que permitirá a las organizaciones educativas proponer planes de capacitación focalizados que permitan fortalecer las capacidades de su personal, además de posicionar a la institución.

Problemática. ¿Cómo un sistema de evaluación del desempeño permite mejorar la posición competitiva de una empresa en Tacna?

Objetivo. Desarrollar un sistema de evaluación del desempeño que permita mejorar la posición competitiva de una empresa en Tacna.

Metodología. Investigación cualitativa. Por ser un trabajo descriptivo no requiere hipótesis.

Variables e indicadores. El desarrollo del presente artículo considera como variable la "Evaluación del Desempeño Laboral"; por tanto, para su análisis se han considerado los siguientes indicadores, (los cuales fueron obtenidos del Test de Dirección por Hábitos) ${ }^{5}$ los cuales se basan en la autoevaluación:

- Perspectiva: Implica tener la visión clara sobre cómo debe evolucionar el trabajo de mi área, y qué debo hacer para situarla en esa meta.

- Equidad: Implica tomar decisiones considerando el poner por encima de los objetivos de mi área los de la empresa.

- Fortaleza: Implica que al presentarse una dificultad me mantengo en la lucha y busco alternativas de solución.

- Equilibrio: Implica respetar el tiempo y el espacio de los demás y procurar no interrumpir con frecuencia a los compañeros.

Procesamiento y técnicas de análisis de datos. Los datos se procesaron a través del software estadístico SPSS versión 18.0; las técnicas de análisis de los datos fueron las siguientes:

${ }^{5}$ http://www.editdiazdesantos.com/wwwdat/pdf/97 88479788636.pdf
- Tablas de frecuencia y de contingencia.

-Medidas de tendencia central (promedio y mediana) y de dispersión (desviación típica).

- Diagramas de caja.

- Estadístico t de Student.

Justificación de la investigación. El procedimiento para evaluar el recurso humano se denomina comúnmente evaluación de desempeño, y generalmente, se elabora a partir de programas formales de evaluación, basados en una razonable cantidad de información respecto de los empleados y de su desempeño en el cargo.

La finalidad del sistema de evaluación de desempeño es orientar la actuación técnica y/o profesional con el objetivo de conseguir la mayor adecuación posible de las personas en sus puestos de trabajo, como medio para obtener su óptimo rendimiento y satisfacción. La mayor parte de los empleados procuran obtener retroalimentación sobre la manera en que se cumplen sus actividades, y las personas que tienen a su cargo la dirección de otros empleados deben evaluar el desempeño individual para decidir las acciones que deben tomar(Solana, 2001).

La evaluación del desempeño es la forma más usada para estimar o apreciar el desenvolvimiento del individuo en el cargo y su potencial de desarrollo; proporciona información valiosa sobre el rendimiento de los trabajadores, permitiendo: la vinculación de la persona al cargo, entrenamiento, promociones, incentivos por el buen desempeño, mejoramiento de las relaciones humanas entre el superior y los subordinados, informaciones básicas para la investigación de los recursos humanos, estimación del potencial de desarrollo de los empleados, estímulo a la mayor productividad.

Limitaciones de la investigación. Al momento de solicitar la información se tuvo poco acceso a dicha información por parte de las entidades regionales (que generó que la investigación se centre en una empresa), bibliografía actualizada escasa relacionada al tema, las facilidades de la entidad para el desarrollo del trabajo de campo según disponibilidad del personal. 


\section{DESARROLLO TEMÁTICO}

\section{Evaluación del desempeño laboral}

La evaluación del desempeño no es un fin en sí mismo, sino un instrumento, una herramienta para mejorar los resultados de los recursos humanos de la empresa. Los superiores jerárquicos están siempre observando la forma en que los empleados desempeñan sus tareas y se van formando impresiones acerca de su valor relativo para la organización. La mayoría de las organizaciones grandes han creado un programa formal, diseñado para facilitar y estandarizar la evaluación de los empleados; sin embargo, resulta poco trabajada la evaluación a nivel de pequeña y mediana empresa. ${ }^{6}$

Muchos son los autores que plantean su concepción acerca de lo que significa la evaluación del desempeño; a continuación, se destacan los siguientes.

Chiavenato (2007) define la evaluación del desempeño como "una apreciación sistemática del desempeño de cada persona en el cargo o del potencial de desarrollo futuro". También agrega que toda evaluación es un proceso para estimular o juzgar el valor, la excelencia y las cualidades de una persona.

Mondy (2005) menciona que, es un procedimiento consecuente de investigación y valoración del desempeño laboral particular o por equipos. La perspectiva de la valoración del desempeño en la totalidad de las empresas se basa en el trabajador en particular. Sin relacionarse al énfasis, el procedimiento de valoración debe evaluar los resultados e iniciar procedimientos de perfeccionamiento, metas y objetivos.

Byars y Rue (1996) indican que la evaluación del desempeño "Es un proceso destinado a determinar y comunicar a los empleados la forma en que están desempeñando su trabajo y, en principio a elaborar planes de mejora". La mejora permitirá a la organización adaptarse a los cambios que se puedan presentar y estar

${ }^{6} \mathrm{http}: / /$ rcientificas.uninorte.edu.co/index.php/pensa miento/article/view/1701/3866 preparados para los nuevos desafíos, sabiendo cómo se encuentra el recurso humano en distintas áreas de la organización.

Harper y Lynch (1992) plantean que es "Una técnica o procedimiento que pretende apreciar, de la forma más sistemática y objetiva posible, el rendimiento de los empleados de una organización. Esta evaluación se realiza en base a los objetivos planteados, las responsabilidades asumidas y las características personales". Evaluar de forma objetiva el rendimiento de los empleados, conforme a los objetivos estratégicos que la organización establece es quizás el reto que tienen los empleados como también los directivos ya que las evaluaciones de desempeño enmarcan la productividad de los empleados como los resultados que los directivos esperan alcanzar para satisfacer a los accionistas o dueños de la organización.

\section{Análisis comparativo}

Con esta comparación se pretende realizar un análisis de los conceptos mencionados por los autores. En efecto, Byars y Rue (1996) mencionan que el desempeño laboral es un proceso destinado a determinar y comunicar a los empleados la forma en que están desempeñando su trabajo $\mathrm{y}$, en principio, a elaborar planes de mejora que ayudarán a realizar de forma eficiente el trabajo; por su parte, Mondy (2005) señala que es una valoración del desempeño laboral de forma particular. Los tres autores tienen distintas formas de definir el desempeño laboral y sus conceptos están vigentes, pero nos quedaremos con Chiavenato (2007), quien define la evaluación del desempeño como una apreciación sistemática del desempeño de cada persona en el cargo o del potencial de desarrollo futuro, agregando que toda evaluación es un proceso para estimular, motivar o juzgar el valor, la excelencia y las cualidades de una persona. Este último autor a diferencia de los anteriores precisa que la evaluación de desempeño es sistemática, tomando en cuenta las particularidades del talento individual de los trabajadores de una organización.

Por tanto, del análisis de los diferentes conceptos, se entiende que la esencia de todo 
sistema de evaluación del desempeño es realizar una valoración, lo más objetiva posible, acerca de la actuación y resultados obtenidos por la persona en el desempeño diario de su trabajo; poniéndose de manifiesto la óptica de la evaluación, la cual, puede decirse, tiene carácter histórico (hacia atrás) y prospectivo (hacia delante), y pretende integrar en mayor grado los objetivos organizacionales con los individuales.

A continuación, se presenta la información estadística de la propuesta del sistema de autoevaluación de desempeño laboral, con sus indicadores respectivos: la perspectiva, la equidad, la fortaleza y el equilibrio.

Tabla 1

Autoevaluación del desempeño laboral

Estadísticos descriptivos

\begin{tabular}{|l|c|c|c|}
\hline & N & Media & Desv. Tip. \\
\hline Desempeño Laboral & 27 & 161.56 & 24.197 \\
\hline
\end{tabular}

\begin{tabular}{|c|c|c|c|c|}
\hline & & Gerencia & & Estadístico \\
\hline & & Media & & 166.55 \\
\hline & & Intervalo de & Límite inferior & 157.16 \\
\hline & Operaciones & la media al $95 \%$ & Límite superior & r 175.93 \\
\hline$\frac{\pi}{8}$ & & Desv. Tip. & & 13.974 \\
\hline 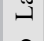 & & Media & & 165.86 \\
\hline : & Servicio & Intervalo de & Límite inferior & 150.36 \\
\hline हू & Académico & la media al $95 \%$ & Límite superior & r 181.36 \\
\hline & & Desv. Tip. & & 16.757 \\
\hline & & Media & & 152.11 \\
\hline & Marketino & Intervalo de & Límite inferior & 124.42 \\
\hline & & la media al $95 \%$ & Límite superior & r 17.80 \\
\hline & & Desv. Tip. & & 36.019 \\
\hline & & Sexo & & Estadístico \\
\hline & & Media & & 158.88 \\
\hline & Masculino & Intervalo de & Límite inferior & 141.48 \\
\hline : & tvase tintu & la media al $95 \%$ & Límite superior & 176.27 \\
\hline हี & & Desv. Tip. & & 20.801 \\
\hline คั & & Media & & 162.68 \\
\hline 퓽 & Femenino & $\begin{array}{l}\text { Intervalo de } \\
\text { confianza para }\end{array}$ & Límite inferior & 150.18 \\
\hline స్తి & & la media al $95 \%$ & Límite superior & 175.19 \\
\hline & & Desv. Tip. & & 25.940 \\
\hline
\end{tabular}

Fuente: Test de autoevaluación del desempeño laboral

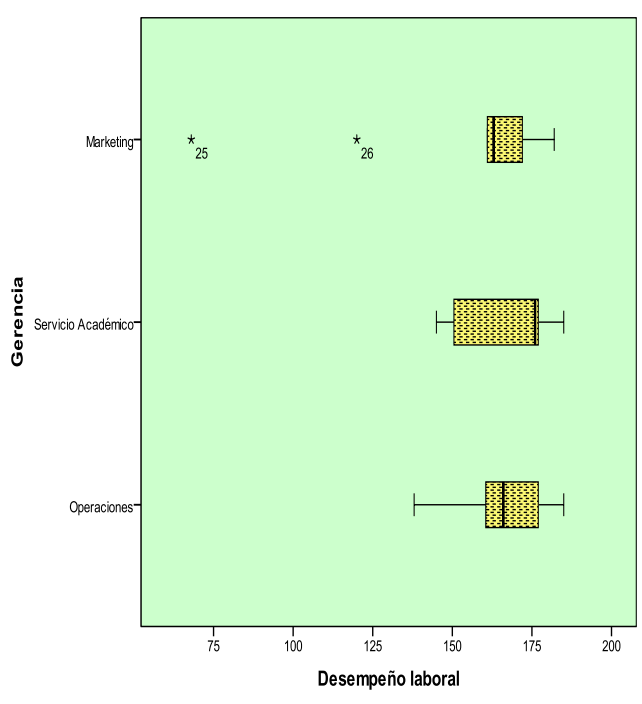

Figura 1: Autoevaluación del desempeño laboral (porgerencia).

Fuente: Test de Autoevaluación del Desempeño Laboral

\section{ANÁLISIS}

Después de efectuar un análisis de cada uno de los indicadores de la autoevaluación del desempeño laboral del personal de la Escuela de Negocios John Von Neumann SRL de Tacna, se procede a analizar los resultados globales, donde el valor medio general fue de 161.56, con una desviación típica de 24.197; dicho valor al ser situado en la escala de valoración determinada, se ubica en la zona definida como "Desempeño laboral adecuado"; por lo tanto, el personal de la entidad autocalifica su desempeño como adecuado o aceptable.

Respecto a la gerencia, se encontró que el valor más elevado se ubicó en la Gerencia de Operaciones, seguida por la Gerencia de Servicio Académico y por la Gerencia de Marketing.

Al analizar por sexo del trabajador, se encontró que son las mujeres las que se autocalifican mejor respecto a los hombres.

Al analizar por indicador de la variable, se encontró que "Equidad" fue el más destacado, seguido por "Perspectiva", "Equilibrio" y "Fortaleza". 


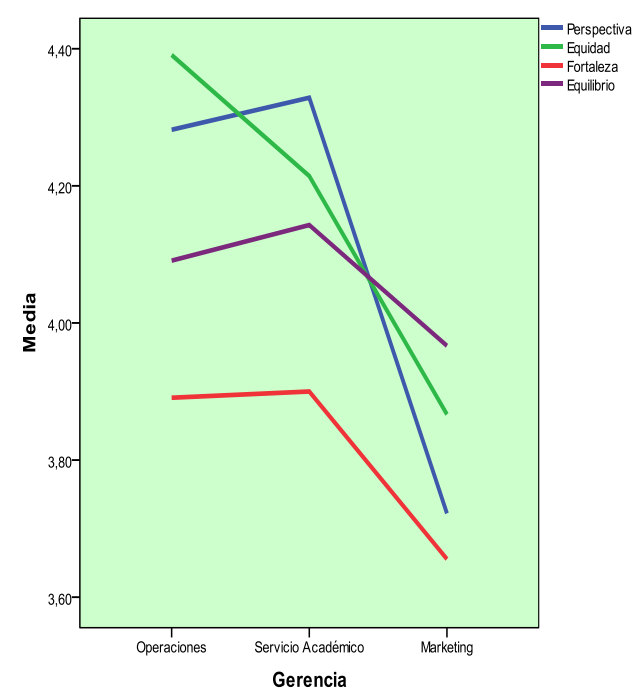

Figura 2: Autoevaluación del desempeño laboral (por indicadores)

Fuente: Test de Autoevaluación del Desempeño Laboral

\section{PROPUESTA DE UN SISTEMA DE EVALUACIÓN DEL DESEMPEÑO LABORAL}

La presente propuesta se centra en la evaluación del desempeño basada en competencias genéricas que deberían alcanzar el personal de la entidad para mejorar su nivel de competitividad y posicionamiento regional; además de resaltar la importancia de apreciar al personal, así como las políticas que deberían servir de guía al momento de medir el desempeño de su personal. Este sistema de evaluación podrá servir y ser aplicado para cualquier escuela de educación en negocios o relacionado con el sector que brinda servicios académicos. Permitiendo impulsar la mejora del nivel de competitividad en la ciudad de Tacna; presentaremos las instrucciones de uso del modelo de evaluación del desempeño y los factores que identifica nuestra propuesta:

\section{Instrucciones de uso del modelo de evaluación del desempeño laboral}

\section{a) Para su uso}

El Modelo de Evaluación del Desempeño Laboral está diseñado para ser aplicado en las áreas de jefaturas y personal administrativo de la Escuela de Negocios John Von Neumann SRL de Tacna y servir como documento de consulta para evaluaciones efectivas.

\section{b) Para su difusión}

El presente documento responde a características propias de la entidad, por tanto, la Gerencia General, debe proceder a la difusión e implementación de la propuesta.

\section{c) Para su mantenimiento}

La propuesta de evaluación de desempeño laboral, deberá ser revisado y actualizado por la Gerencia General por lo menos una vez al año, según las necesidades de la institución o por el surgimiento de información relacionada con el mismo.

\section{Factores identificados}

Conocimiento del trabajo: Se refiere al conocimiento que tienen los trabajadores administrativos con respecto a sus puestos de trabajo, habilidades y destrezas para desarrollarlo de manera eficiente tomando en consideración el manual de funciones. Los criterios a evaluar dentro de este factor serán:

- Competente en habilidades y conocimientos requeridos para el trabajo.

- Muestra habilidad para aprender y aplicar nuevas técnicas.

- Muestra interés por mantener informes de avances propios de su cargo.

\section{Tabla 2}

Factor del conocimiento del trabajo

$\begin{array}{lc}\text { Conocimiento del trabajo } & \text { Puntos } \\ \text { Necesita mejorar } & 1 \\ \text { Ocasionalmente necesita ayuda } & 2 \\ \text { Cumple con los requerimientos exigidos } & 3 \\ \text { Demuestra conocimiento en su puesto } & 4 \\ \text { Sobresaliente } & 5\end{array}$

Calidad de trabajo: En este factor se evalúa la capacidad del trabajador administrativo en la producción de un servicio eficiente, oportuno y preciso a los usuarios de la entidad. Los criterios a evaluar dentro de este factor serán: 
- Demuestra exactitud e integridad.

- Muestra compromiso hacia la excelencia.

- Busca formas para mejorar y promover la calidad de su trabajo.

- Aplica retroalimentación para mejorar su desempeño laboral.

- Revisa su trabajo para asegurar la calidad del mismo.

Dichos criterios serán calificados en base a puntos, los cuales tendrán el siguiente valor y su respectivo significado:

Tabla 3

Factor de calidad del trabajo

$\begin{array}{|lc|}\text { Calidad de trabajo } & \text { Puntos } \\ \text { Muy deficiente } & 1 \\ \text { Comete pocos errores } & 2 \\ \text { Calidad de trabajo aceptable } & 3 \\ \text { Por encima del rendimiento promedio } & 4 \\ \text { Sobresaliente } & 5\end{array}$

Eficiencia / Productividad: Es el logro de los objetivos con la mínima cantidad de recursos. En este factor se evalúa la medida del desempeño del personal y la capacidad que tienen para el buen manejo de los recursos disponibles para lograr los objetivos propuestos. Los criterios a evaluar son:

- Cumple los estándares de productividad.

- Completa su trabajo en el tiempo estipulado.

- Se esfuerza por incrementar su productividad.

- Trabaja rápidamente.

- Logra las metas establecidas.

La forma de evaluación se detalla a continuación:

Tabla 4

Factor de eficiencia/productividad

\begin{tabular}{lc} 
Eficiencia / Productividad & Puntos \\
\hline Rendimiento no satisfactorio & 1 \\
Necesita mejorar & 2 \\
Su rendimiento es aceptable & 3 \\
Excede los estándares requeridos & 4 \\
Es muy cuidadoso, exacto y & 5 \\
sobresaliente &
\end{tabular}

Actitud e iniciativa: Son los juicios favorables o desfavorables que el trabajador tiene con respecto a su puesto de trabajo, así como la capacidad para diseñar, proponer y ejecutar innovaciones en el desarrollo de las funciones de su puesto de trabajo. Los criterios que se evaluarán dentro de este factor son:

- Establece y mantiene efectivas relaciones de trabajo con sus compañeros.

- Realiza su trabajo con entusiasmo y buena disposición.

- Trabaja en equipo, ofrece ayuda y colaboración a sus compañeros de trabajo.

- Acepta con agrado tareas no asignadas comúnmente a su puesto.

- Busca incrementar las responsabilidades asignadas.

- Busca ayuda oportuna cuando se requiere.

Se evaluará mediante puntuaciones que se detallan a continuación:

Tabla 5

Factor de actitud e iniciativa

\begin{tabular}{|c|c|}
\hline Actitud e iniciativa & Punto \\
\hline Carece totalmente de iniciativa & 1 \\
\hline $\begin{array}{l}\text { Solo realiza trabajos que se le } \\
\text { encomiendan }\end{array}$ & 2 \\
\hline $\begin{array}{l}\text { Ocasionalmente realiza tareas fuera de } \\
\text { lo encomendado }\end{array}$ & 3 \\
\hline $\begin{array}{l}\text { Frecuentemente realiza tareas y } \\
\text { actividades por iniciativa propia }\end{array}$ & 4 \\
\hline $\begin{array}{l}\text { Siempre pone en práctica su actitud e } \\
\text { iniciativa }\end{array}$ & 5 \\
\hline
\end{tabular}

Atención al cliente y responsabilidad: Es el arte de tener buenas relaciones interpersonales con clientes internos y clientes externos. Estos criterios serán calificados en base a puntos, los cuales tendrán el siguiente valor y su respectivo significado:

- Mantiene armonía laboral con su jefe y compañeros.

- Se esfuerza por brindar buena atención al público.

- Es puntual y responsable con su trabajo. 
- Muestra confianza y amabilidad al momento de brindar servicios al cliente.

- Muestra lealtad hacia la institución y honestidad con el cliente.

La forma de evaluación se detalla a continuación:

Tabla 6

Factor atención al cliente y responsabilidad

Atención al cliente
y responsabilidad

No satisface

Pocas veces

1

A veces

2

Frecuentemente

Siempre

Solución de problemas: Capacidad de corregir situaciones que se presentan, cuando el estado real de las cosas no es igual al estado deseado de las mismas. Criterios a evaluar:

- Identifica y previene problemas a tiempo.

- Consolida y analiza la información de forma efectiva.

- Desarrolla alternativas de solución.

- Resuelve problemas en etapas tempranas.

- Trabaja muy bien en grupo en situaciones de solución de problemas.

Los valores asignados para la evaluación de estos criterios se presentan en la siguiente tabla:

\section{Tabla 7}

Factor solución de problemas

\begin{tabular}{|lc} 
Solución de problemas & Puntos \\
\hline Carece de capacidad & 1 \\
Ocasionalmente muestra iniciativa & 2 \\
Su capacidad de proceder es aceptable & 3 \\
Cumple las expectativas & 4 \\
Sobresaliente & 5
\end{tabular}

Planificación y organización del trabajo: Se refiere a la estructura y organización del trabajo, los procedimientos a seguir para desarrollar las funciones y tareas del puesto, a fin de lograr los objetivos establecidos. Los

criterios a considerar en este ítem son:

- Prioriza y planifica sus actividades de trabajo.

- Usa efectivamente el tiempo.

- Distribuye las labores adecuadamente.

- Integra cambios efectivamente.

- Comunica oportunamente la información.

- Trabaja de forma organizada.

Se evaluará mediante puntuaciones que se detallan a continuación:

Tabla 8

Factor planificación y organización del trabajo

Planificación y organización
del trabajo

Desempeño insatisfactorio 1

Ocasionalmente no cumple con lo requerido 2

Cumple con los requisitos de trabajo 3

Cumple y excede lo requerido por el puesto 4

Supera las expectativas del puesto

Toma de decisiones: Se entenderá por toma de decisiones a la selección de un curso de acción ante varias alternativas. Constituye, por lo tanto en la esencia de la planeación. Los criterios a evaluar son:

- Muestra habilidad para tomar decisiones.

- Expone criterios sólidos y acertados.

- Sostiene y explica razonamiento para la toma de decisiones.

- Involucra apropiadamente, a su personal, el proceso de toma de decisiones.

- Toma decisiones de forma oportuna.

La forma de evaluación se detalla a continuación:

Tabla 9

Factor toma de decisiones

Toma de decisiones

Deficiente

Pocas veces

Frecuentemente

Casi siempre

Siempre
Puntos

1

4

5 
Liderazgo y trabajo en equipo: Es la capacidad de influir en las demás personas para que realicen voluntariamente actividades que conllevaran a lograr los objetivos deseados. Los criterios a evaluar son:

- Denota confianza en sí mismo y sobre los demás.

- Inspira respeto y confianza.

- Reacciona muy bien bajo presión.

- Muestra determinación para ejecutar acciones.

- Motiva a otros hacia un buen desempeño.

- Mantiene armonía laboral con su jefe y compañeros.

Los valores asignados para la evaluación son:

Tabla 10

Factor toma de decisiones

\begin{tabular}{|lc|}
\hline Liderazgo y trabajo en equipo & Puntos \\
\hline Necesita mejorar & 1 \\
Pocas veces & 2 \\
Frecuentemente & 3 \\
Excede lo esperado & 4 \\
Sobresaliente & 5 \\
\hline
\end{tabular}

Entrenamiento y capacitación del personal:

Se refiere al adiestramiento y reforzamiento de las funciones del personal, por medio de la práctica y conocimientos básicos que contribuyan a lograr una mayor efectividad de las funciones. Los criterios son:

- Forma buenos empleados, enseña y asesora.

- Detecta e informa a tiempo las necesidades de entrenamiento del personal.

- Coordina y/o participa en las tareas de entrenamiento asignadas.

- Brinda apoyo a las actividades de capacitación.

- Motiva hacia el aprendizaje continuo.

Se evaluará mediante puntuaciones que se detallan a continuación:
Tabla 11

Factor entrenamiento y capacitación de su personal

\begin{tabular}{|lc|}
$\begin{array}{l}\text { Entrenamiento y capacitación } \\
\text { de su personal }\end{array}$ & Puntos \\
\hline No satisface & 1 \\
Pocas veces & 2 \\
Frecuentemente & 3 \\
Excede lo esperado & 4 \\
Sobresaliente & 5
\end{tabular}

Tabla de rangos y puntajes de la evaluación:

Después de los puntajes determinados para cada uno de los 10 factores considerados en la evaluación del puesto de trabajo respectivo, se procede a multiplicar dichos valores por los "Pesos o relevancia relativa", previamente definidos por el evaluador, los cuales deben ubicarse en la siguiente tabla de rangos, con la finalidad de determinar el desempeño laboral del personal.

Tabla 12

Rangos para evaluar el desempeño laboral

\begin{tabular}{|c|c|}
\hline Escala de Puntos & $\begin{array}{l}\text { Nivel de desempeño } \\
\text { laboral }\end{array}$ \\
\hline De 1.00 a 1.74 puntos & No satisfactorio (bajo) \\
\hline De 1.75 a 2.74 puntos & Necesita mejorar \\
\hline De 2.75 a 3.74 puntos & $\begin{array}{l}\text { Cumple los requerimientos } \\
\text { de trabajo (promedio) }\end{array}$ \\
\hline De 3.75 a 4.74 puntos & $\begin{array}{l}\text { Cumple y excede los } \\
\text { requerimientos del puesto }\end{array}$ \\
\hline De 4.75 a 5.00 puntos & Sobresaliente (alto) \\
\hline
\end{tabular}

Indicaciones: En el caso que, el personal cuyo puntaje esté comprendido en los niveles denominados "No satisfactorio" y "Necesita mejorar", la entidad debe priorizar en ellos la focalización del Plan de Capacitación de fortalecimiento de Competencias Genéricas.

Por otro lado, en el caso que, el personal cuyo puntaje esté comprendido en los niveles denominados "Cumple y excede los requerimientos del puesto" y "Sobresaliente", la entidad debe aprovechar dicho desempeño que permita ser ejemplo para los demás colaboradores, y genere el proponer una política de incentivos y ascensos en la entidad. 
Si se da el caso que, el personal cuyo puntaje esté comprendido en el nivel "Cumple los requerimientos de trabajo", la entidad debe desarrollar en ellos técnicas de motivación y de impulso al compromiso organizacional, que impulse el alcanzar un desempeño sobresaliente.

\section{RETOS Y OPORTUNIDADES}

La mayoría de empresas buscan obtener resultados económicos (rentabilidad) a corto plazo, ya que la competencia es en realidad una variable que suele sacar del mercado a sus rivales. Sin embargo, se debe tener en cuenta que un aspecto importante en las organizaciones no es quien logra mantenerse en el mercado o quien logra obtener mejores resultados al final de sus ejercicios comerciales, sino quien logra consolidar un equipo de desempeño laboral excelente, que logre ser en el mercado un factor determinante de ventaja competitiva; siendo esta una labor de mediano y largo plazo.

Las empresas que brindan servicios académicos necesitan formar, capacitar y consolidar un equipo de trabajo que pueda responder a las exigencias y objetivos que la empresa y el mercado necesitan, evaluar el nivel de desempeño laboral será la manera de no dejar pasar la oportunidad de mejorar frente a las dificultades y deficiencias que pudiera tener el personal; son los retos de las organizaciones que brindan servicios académicos tienen en el sector.

Es por eso que esta propuesta de Sistema de Evaluación de Desempeño Laboral, es una gran oportunidad para fortalecer las competencias y consolidarlas en la dinámica de actividades laborales.

Los beneficios esperados para las organizaciones al implementar la propuesta de un modelo para la evaluación del desempeño laboral se relacionan con conseguir que su personal se encuentre más comprometido, logre un mayor nivel de satisfacción laboral, fomente a fortalecer el trabajo en equipo, una mejor comunicación organizacional, entre otros; puesto que cada uno de los colaboradores tienen claro en qué aspectos de su desempeño laboral deben darle principal atención, puesto que se tratan de competencias que la Gerencia o Dirección tiene la expectativa que se efectué desempeños positivos dependiendo del cargo del colaborador; además le permitirá a los directivos proponer planes de capacitación focalizados que permitan fortalecer las capacidades de su personal, las rotaciones respectivas o los cambios que estime por conveniente.

\section{CONCLUSIONES}

El presente artículo se centró en evaluar el desempeño laboral de las empresas que brindan servicios académicos, y en este caso se evaluó a la Escuela de Negocios John Von Neumann SRL de Tacna, basando el trabajo de campo en el total de trabajadores administrativos; es decir 27 personas, las cuales fueron agrupadas en: Gerencia de Operaciones, Gerencia de Servicio Académico y Gerencia de Marketing.

Se aplicó un test de autoevaluación del desempeño laboral, lo cual permite concluir lo siguiente: El personal de la Escuela de Negocios John Von Neumann SRL de Tacna autocalifica su desempeño como adecuado o aceptable, complementando ello a través del análisis por indicadores.

Se concluye de forma específica, que la "Equidad" es el más destacado, seguido por la "Perspectiva", por el "Equilibrio" y por la "Fortaleza"; de donde:

- El indicador "Perspectiva" tuvo una media de 4.11, que implica que la autoevaluación en este aspecto fue "muy adecuada". Siendo el aspecto a mejorar: El involucrarse más con el trabajo de los compañeros y conocer sus metas personales y profesionales.

- El indicador "Equidad" tuvo una media de 4.17, que implica que la autoevaluación en este aspecto fue "muy adecuada". Siendo el aspecto a mejorar: El impulsar la crítica constructiva que permita intercambiar puntos de vista entre los compañeros.

- El indicador "Fortaleza" tuvo una media de 3.81, que implica que la autoevaluación en este aspecto fue "adecuada". Siendo el aspecto a mejorar: Ser más empático, poniendo más atención a los problemas de los demás antes que a los propios.

- El indicador "Equilibrio" tuvo una media de 
4.06, que implica que la autoevaluación en este aspecto fue "muy adecuada". Siendo el aspecto a mejorar: El respetar el tiempo y el espacio de los demás.

En base a los resultados de la autoevaluación de desempeño y a las coordinaciones con la Alta Dirección de la entidad, se desarrolló la propuesta de un modelo para la evaluación del desempeño que pueda ser utilizada o aplicada para cualquier empresa que brinde servicios académicos. Basado en un conjunto de competencias generales para un desempeño laboral destacado. Dichos factores de evaluación son:

- Conocimiento del trabajo.

- Calidad de trabajo.

- Eficiencia/Productividad.

- Actitud e iniciativa.

- Atención al cliente y responsabilidad.

- Solución de problemas.

- Planificación y organización del trabajo.

- Toma de decisiones.

- Liderazgo y trabajo en equipo.

- Entrenamiento y capacitación del personal.

Por tanto, se concluye que las empresas que brinden servicios académicos deben considerar el implementar esta propuesta de evaluación, lo cual permitirá precisar las competencias genéricas a reforzar, a través de planes de capacitación focalizados que permitan fortalecer las capacidades de su personal logrando con ello un desempeño destacado o excelente, las decisiones de forma objetiva que deba de tomar en torno a las rotaciones respectivas de personal o los cambios que estime por conveniente en la organización.

\section{REFERENCIAS BIBLIOGRÁFICAS}

Byars, L. y Rue, L. (1996). Gestión de Recursos Humanos. España: Editorial Mosby, Ediciones División IRWIN.

Chiavenato, I. (2007). Administración de recursos humanos. México: Editorial McGraw Hill/lnteramericana Editores S.A.

Harper y Lynch (1992). Manuales de Recursos Humanos. Madrid, España: Editorial Gaceta de Negocios.

Mondy, R. y Robert, N. (2005). Administración de recursos humanos. México: Editorial Pearson Educación.

Solana, R. (2001). Administración de organizaciones. Buenos Aires, Argentina: Ediciones Interoceánicas S.A. 